\title{
Neurocysticercosis in a patient in Canada
}

\author{
Jorge G. Burneo MD MSPH, lan Plener BSc, Hector H. Garcia MD PhD, Neurocysticercosis \\ and Epilepsy Research Network
}

A 51-year-old man presented with a single episode of generalized tonic-clonic seizure after returning to Canada from a trip to Mexico. A farmer, he was originally from Mexico and had lived in Canada for several years. He reported no weight loss or other symptoms of concern before the seizure. He had a history of heavy smoking. He had worked with pigs for several months in the past but had not been exposed to them recently. A physical examination was normal and showed no neurologic deficits.

Laboratory tests showed mild leukocytosis without eosinophilia, most likely related to the seizure. A computed tomography scan of the head and a subsequent magnetic resonance imaging scan of the brain showed 2 cystic lesions in the left frontal area. One lesion was located in the left inferior frontal gyrus and measured $1.2 \mathrm{~cm}$ in its largest diameter with significant perilesional edema (Figure 1A). The other was a larger lesion in the left superior and middle frontal lobe measuring $3 \mathrm{~cm}$ (Figures $1 \mathrm{~B}$ and $1 \mathrm{C}$ ). Because of the patient's extensive history of smoking, the initial working diagnosis was metastatic lung cancer. However, the metastatic workup, including computed tomography scans of the chest and abdomen, was negative.

One of the lesions was surgically removed without complications. The postoperative pathologic diagnosis was neurocysticercosis. The patient was transferred to the neurology service and given albendazole $400 \mathrm{mg}$ twice daily for 10 days, dexamethasone $4 \mathrm{mg} 3$ times daily for 7 days and phenytoin $300 \mathrm{mg}$ daily. At a 3-month follow-up visit, the patient was still taking phenytoin and had had no recurrence of seizures. A computed tomography scan of the head showed that the lesions had resolved. At a 6-month follow-up visit, he continued to be seizure free. Phenytoin was discontinued without recurrence of seizures.

\section{Discussion}

Neurocysticercosis is the most common parasitic disease of the nervous system. It accounts for about 50000 deaths per year and many times this number of people with active epilepsy. ${ }^{1,2}$ In disease-endemic areas (Figure 2), about a third of all cases of epilepsy are due to neurocysticercosis. ${ }^{3}$ With increased travel to disease-endemic areas and the migration of tapeworm carriers or people infected with the disease, neurocysticercosis is becoming increasingly prevalent in industrialized countries. ${ }^{4}$ Neurocysticercosis is occasionally seen in Canada.

\section{Key points}

- Neurocysticercosis is the most common parasitic disease of the central nervous system and is linked to accidental ingestion of pork tapeworm eggs.

- It causes about 50000 deaths per year and is the leading cause of epilepsy worldwide.

- Prevalence of infection is increasing in developed countries as more people travel to or migrate from regions where infection is endemic.

- Common neurologic symptoms include late-onset epilepsy, headache, focal signs and dementia.

- Patients with suspected neurocysticercosis should be given anticysticidal drugs and followed up with neuroimaging studies before surgical resection is considered.

\section{Life cycle}

Cysticercosis results from the accidental ingestion of Taenia solium (pork tapeworm) eggs, usually from food contaminated by people with taeniasis. The eggs of $T$. solium can remain viable for up to 2 months in water, soil or vegetation. Once the eggs are in the intestinal tract, the actions of bile and pancreatic enzymes dissolve their protective coatings. Liberated from their coats, they become embryos (oncospheres), cross the intestinal wall and enter the bloodstream. There they are carried to the tissues of the host, including the brain, and become established as cystic larvae (cysticerci).

In some areas of the world, particularly those with deficient disposal of human feces, pigs serve as scavengers, consuming human feces that often contain $T$. solium eggs. In these instances, pigs become the intermediate hosts in the life cycle of the tapeworm. Taeniosis in humans results from consumption of improperly cooked meat from swine that are infected by cysticerci. The condition is usually benign, although it may produce abdominal discomfort and peripheral eosinophilia.

Some cysticerci are rejected as they enter the tissues of a human host. From the moment a cysticercus invades the brain tissue, for example, it is exposed to a hostile environment. When the host's immune system recognizes the parasite as foreign, it usually mounts an appropriate inflammatory reac-

From the Epilepsy Program (Burneo), London Health Sciences Centre, University of Western Ontario, London, Ont.; the School of Medicine (Plener), Queen's University, Kingston, Ont.; and Universidad Peruana Cayetano Heredia, Instituto de Ciencias Neurológicas and Cysticercosis Working Group in Peru (Garcia), Lima, Peru 

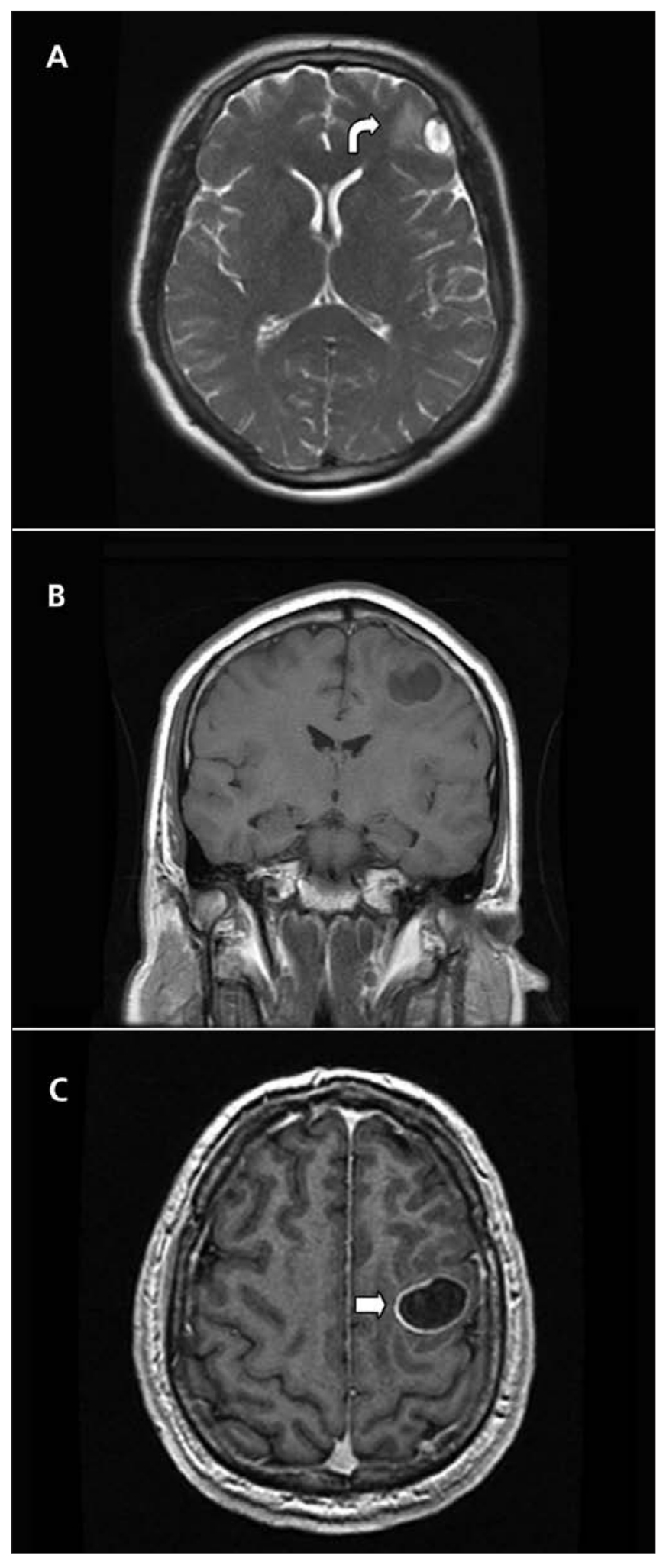

Figure 1: Magnetic resonance imaging scans of the head of a 51-year-old man with neurocysticercosis, showing $\left(A, T_{2^{-}}\right.$ weighted coronal view) a $1.2-\mathrm{cm}$ lesion in the left inferior frontal gyrus with surrounding edema (arrow); ( $B, T_{1}$-weighted axial view) a $3-\mathrm{cm}$ lesion in the left superior and middle frontal lobes; and ( $C$, with contrast) ring enhancement of the larger lesion (arrow). tion to overcome the infection. In many cases, however, such a response does not occur, and the host reaches a state of immunologic tolerance to the parasite, leaving it almost undisturbed for many years. No clear explanation exists for the individual differences observed in the severity of immunologic response against infection of the central nervous system by cysticerci. It is unknown why some people experience seizures and others do not.

\section{Clinical presentation}

Neurocysticercosis can cause many neurologic symptoms. Late-onset epilepsy is the most common clinical symptom; neurocysticercosis is the leading cause of epilepsy worldwide. ${ }^{5}$ Patients with neurocysticercosis may also present with headache associated with intracranial hypertension (another common presentation in disease-endemic countries), focal signs or dementia. Intracranial hypertension is most commonly caused by hydrocephalus related to granular ependymitis, arachnoiditis or ventricular cysts. Other causes include giant cysts or cysticercotic encephalitis.

The course of disease may remain subacute or chronic for many years, then present with focal signs of a cerebrovascular event secondary to an acute inflammatory response to the parasites. Cysticerci can also invade the spinal cord, the eyes and the subcutaneous and muscular tissues of the body. Muscular pseudohypertrophy is seen more frequently in patients from Asia who have cysticercosis; it accounts for $0.6 \%$ of cases in China. Muscular pseudohypertrophy is extremely rare in America.

\section{Diagnosis}

Diagnosis of neurocysticercosis is based primarily on results of neuroimaging studies and laboratory tests (Box 1). Computed tomography is the most commonly used neuroimaging test for the disease and maintains relatively good diagnostic sensitivity when used in disease-endemic areas. Calcifications in the brain parenchyma are the most common finding in computed tomography studies and, in many cases, the only radiologic evidence of the disease. For that reason, computed tomography remains the best screening tool for assessing patients with suspected neurocysticercosis.

Multiple lesions in the parenchyma at different stages are commonly seen in neurocysticercosis. Small lesions, especially those situated close to bone or within ventricles, may be missed on computed tomography scans. A magnetic resonance imaging scan is therefore often added for increased diagnostic sensitivity and accuracy. Magnetic resonance imaging is also the modality of choice when evaluating patients with intraventricular cysticercosis and when assessing brainstem cysts and small cysts located over the convexity of the cerebral hemispheres. The main shortcoming of magnetic resonance imaging is its failure to detect small calcifications.

The appearance of cysticerci in brain parenchyma on neuroimaging scans depends on their stage of development. The cysticercus has 4 stages of involution: vesicular, colloidal, granular and calcified. Vesicular cysts appear as rounded lesions with signal properties similar to those of cerebrospinal fluid in both $T_{1}$ - and $T_{2}$-weighted images. The scolex may be 
seen within the cyst as a high-intensity nodule, giving the lesion a pathognomonic "hole-with-dot" appearance (Figure 3). When the parasite begins to degenerate, the lesion becomes heterogeneous, and its appearance varies depending on the degree of degeneration. Cysticercotic encephalitis involves multiple cysts in the brain parenchyma associated with severe, diffuse inflammation.

Cysticercosis may present with a single intraparenchymal brain lesion with nodular or ring enhancement detected with the use of contrast in neuroimaging studies (Figure 1C). The differential diagnosis for this type of lesion includes brain tumours, hydatidosis, multiple sclerosis and tuberculomas. The use of epidemiologic information together with clinical suspicion and laboratory tests is essential in making the final diagnosis and starting treatment.

Hematologic abnormalities in patients with neurocysticercosis include mild leukocytosis and eosinophilia in up to $30 \%$ of patients. Stool examination for $T$. solium eggs is positive in only $5 \%-10 \%$ of patients. In a similar proportion of cases, a $T$. solium carrier can be found in the patient's close environment. Because analysis of cerebrospinal fluid shows abnormalities in $50 \%$ or less of patients with neurocysticercosis, a normal finding on examination of cerebrospinal fluid does not rule out neurocysticercosis. Serologic assays, mainly enzyme-linked immunoelectron transfer blot, are also commonly used to detect specific antibodies. Resolution of intracranial cystic lesions

\section{Box 1: When to consider a diagnosis of} neurocysticercosis

- Cystic lesions, solitary enhancing lesions or punctuate calcifications observed on neuroimaging studies

- Resolution of intracranial cystic lesions after treatment with albendazole or praziquantel

- New-onset seizure in an adult

- Evidence of household contact with Taenia solium infection

- Origination from region where neurocysticercosis is endemic

- History of repeated travel to disease-endemic regions

after treatment with albendazole or praziquantel indicates the presence of neurocysticercosis.

Epidemiologic factors that may lead to a diagnosis of neurocysticercosis include evidence of household contact with $T$. solium, immigration from an area where the disease is endemic and a history of repeated travel to disease-endemic regions. Detection of the parasite in a biopsy of a brain or spinal cord lesion is one of the proposed absolute criteria for the diagnosis of neurocysticercosis. ${ }^{6}$ However, the combination of epidemiologic, clinical and laboratory information may lead to an accurate diagnosis and avoid an invasive diagnostic procedure.

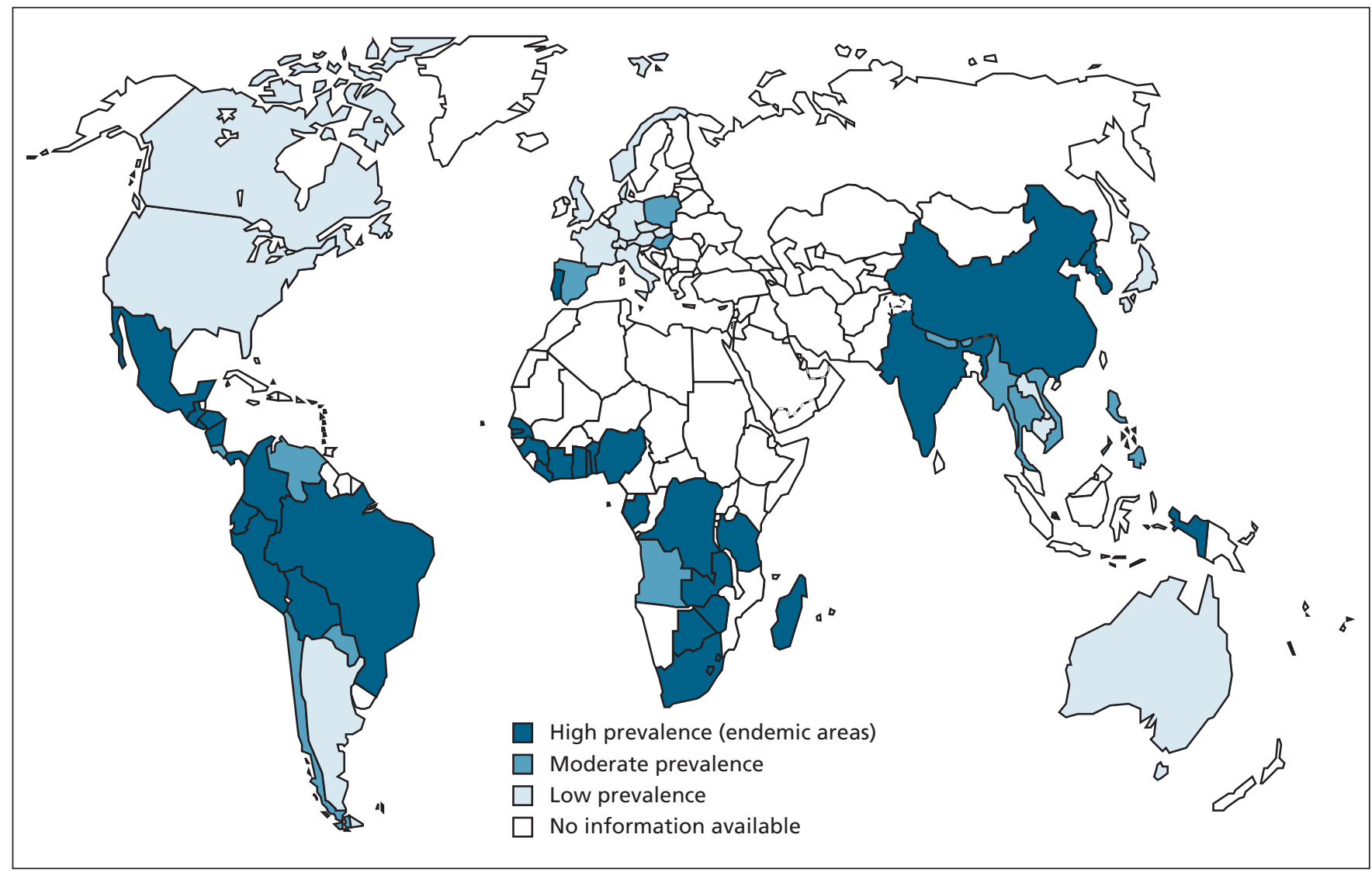

Figure 2: Worldwide prevalence of neurocysticercosis. Reproduced, with permission, from Roman et al. ${ }^{2}$ 


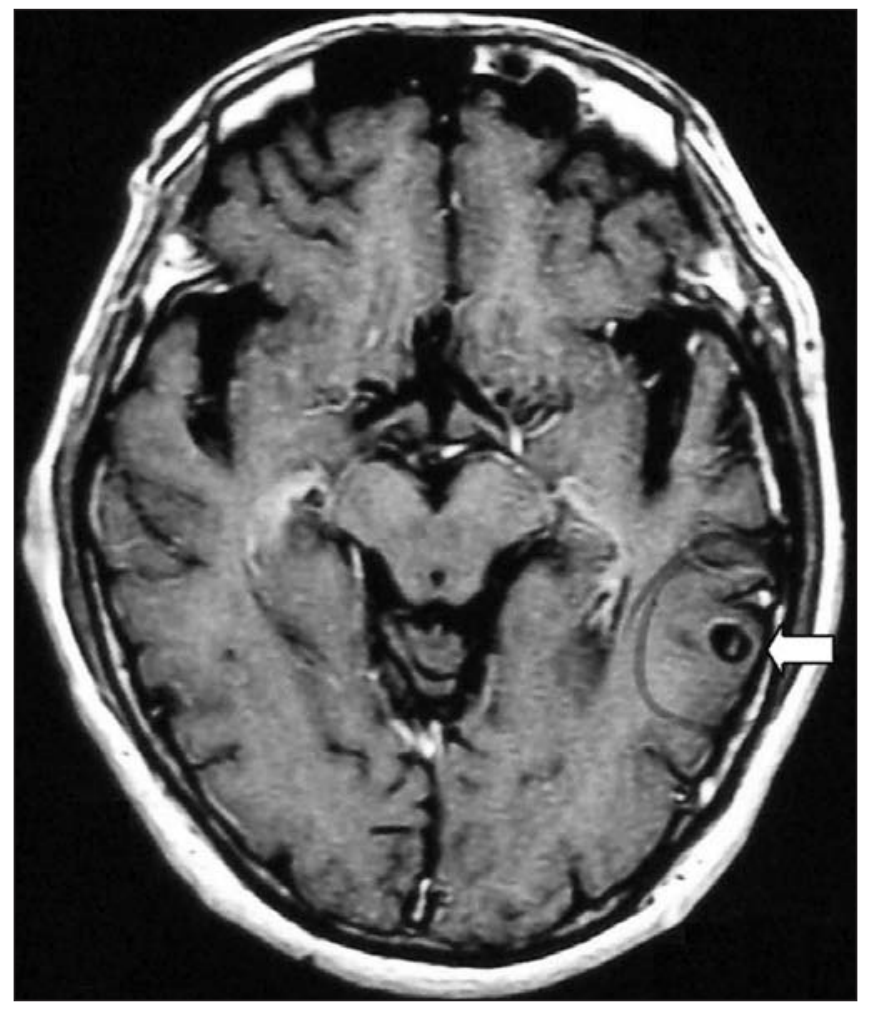

Figure 3: Axial magnetic resonance imaging scan of the brain of a different patient with neurocysticercosis showing a vesicular cyst (arrow). The head of the larva in the cyst gives the lesion a pathognomonic "hole-with-dot" appearance.

\section{Treatment}

A recent meta-analysis ${ }^{7}$ found that complete resolution of cystic lesions occurred in $44 \%$ of patients given specific cysticidal therapy compared with $19 \%$ of patients not given treatment $(p=0.025)$, with better results for viable cysts. The recurrence of seizures was reduced in $14 \%$ of patients given cysticidal therapy compared with $37 \%$ of those not given treatment $(p<0.001)$.

Albendazole and praziquantel are widely accepted cysticidal drugs. Albendazole is used most frequently because of its greater availability, higher efficacy and lower rates of interaction with antiepileptic drugs such as carbamazepine. Both drugs are available in Canada by special access through Health Canada. The standard dose of albendazole is $15 \mathrm{mg} / \mathrm{kg}$ daily for 10-14 days.

Cysticidal drugs damage the parasite and release antigens, triggering an inflammatory reaction that may decompensate the patient. Steroids are therefore used frequently during therapy with anticysticercal drugs in patients with cysticercosis in the brain parenchyma. Clinicians should treat cases of sus- pected neurocysticercosis empirically with anticysticidal drugs and follow up with neuroimaging studies before considering surgical resection. Before the introduction of anticysticercal drugs, surgery was considered a feasible therapeutic approach to neurocysticercosis. Now the role of surgery is limited to shunt placement for the control of hydrocephalus in patients with subarachnoid neurocysticercosis and to the excision of large cysts in the parenchyma in selected patients.

Neurocysticercosis has become an increasingly important emerging infection in the developed world. Its rising prevalence is driven largely by increased migration from diseaseendemic regions and ease of international travel. Widespread access to neuroimaging diagnostic tools has permitted easier diagnosis. A multinational effort is underway to study the epidemiology, etiopathogenesis and treatment of this disease as a cause of epilepsy. New developments in this effort include the recent creation of the Neurocysticercosis and Epilepsy Research Network. ${ }^{8}$

Competing interests: None declared.

\section{REFERENCES}

1. Gemmell MA, Matyas Z, Pawlowsky Z, et al. Guidelines for surveillance, prevention and control of taeniasis/cysticercosis. Geneva (Switzerland): World Health Organization; 1983.

2. Roman G, Sotelo J, Del Brutto O, et al. A proposal to declare neurocysticercosis an international reportable disease. Bull World Health Organ 2000;78:399-406.

3. Nash TE, Pretell EJ, Lescano AG, et al. Perilesional brain oedema and seizure activity in patients with calcified neurocysticercosis: a prospective cohort and nested case-control study. Lancet Neurol 2008;7:1099-105.

4. Wallin MT, Kurtzke JF. Neurocysticercosis in the United States: review of an important emerging infection. Neurology 2004;63:1559-64

5. Garcia HH, Gilman R, Martinez M, et al. Cysticercosis as a major cause of epilepsy in Peru. The Cysticercosis Working Group in Peru (CWG). Lancet 1993; 341:197-200.

6. Del Brutto OH, Rajshekhar V, White AC Jr, et al. Proposed diagnostic criteria for neurocysticercosis. Neurology 2001;57:177-83.

7. Del Brutto OH, Roos KL, Coffey CS, et al. Meta-analysis: cysticidal drugs for neurocysticercosis: albendazole and praziquantel. Ann Intern Med 2006;145:43-51.

8. Burneo JG, Delgado-Escueta A, Gonzalez AE, et al. Developing an international collaborative research network in neurocysticercosis and epilepsy (NERN). Epilepsia. In press.

The section Cases presents brief case reports that convey clear, practical lessons. Preference is given to common presentations of important rare conditions, and important unusual presentations of common problems. Articles start with a brief summary (100 words) outlining the case and its relevance to a general audience. The case presentation follows (500 words maximum) as well as a discussion of the underlying condition (1000 words maximum). Generally, up to 5 references are permitted and visual elements (e.g., tables of the differential diagnosis, clinical features or diagnostic approach) are encouraged. Written consent from patients for publication of their story is a necessity and should accompany submissions. See information for authors at www.cmaj.ca. 